\title{
Prospecção Tecnológica na Indústria de Alimentos e Bebidas: um panorama dos processos e equipamentos para produção de malte
}

\author{
Technological Foresight in the Food and Drink Industry: an overview of \\ the processes and equipments for malt production
}

\author{
Marcio Lima da Silva ${ }^{1}$ \\ Larisse Araújo Lima ${ }^{1}$ \\ Lincoln Pinheiro Oliveira ${ }^{1}$ \\ Luiza Xavier da Silva Tenório ${ }^{1}$ \\ Sarah Sampaio Py-Daniel ${ }^{1}$ \\ Thiago Lara Fernandes ${ }^{1}$ \\ Grace Ferreira Ghesti ${ }^{1}$ \\ ${ }^{1}$ Universidade de Brasília, Brasília, DF, Brasil
}

\begin{abstract}
Resumo
O presente trabalho apresenta uma prospecção tecnológica, aplicada à indústria de alimentos e bebidas, de processos e equipamentos para produção de malte. A metodologia de pesquisa realizou buscas em plataformas de busca de patentes e artigos científicos, usando como palavras-chave malt, malting, brewing, process, production, cereal e grain. Foram encontrados 2.109 patentes e 732 artigos, de 2008 a 2017; sendo 68\% das patentes atribuídas à China e $19 \%$ à Rússia. Em relação aos artigos, a Alemanha apresenta a maior produção com $9,4 \%$ dos artigos, seguida pela Irlanda com $7,2 \%$. A China apresenta um cenário de tecnologia consolidada, com um grande número de documentos patentários e baixo número de artigos científicos. O Brasil está em um cenário de potencial aplicação, porém encontra-se em fase de desenvolvimento tecnológico, mas destacam-se as iniciativas das universidades, ao desenvolverem tecnologias voltadas para esse mercado e realizarem o depósito, conferindo-lhes visibilidade dentro do setor industrial.
\end{abstract}

Palavras-chave: Engenharia de alimentos. Prospecção tecnológica. Produção de malte.

\begin{abstract}
The present work presents a technological prospection, applied to the food and beverage industry, processes and equipment for malting. The research methodology carried out searches on patent search platforms and scientific articles, using malt, malting, brewing, process, production, cereal and grain as keywords. 2109 patents and 732 articles were found between 2008 and 2017; with 68\% of patents attributed to China and 19\% to Russia. In relation to the articles, Germany has the highest production with $9.4 \%$ of articles, followed by Ireland with $7.2 \%$. China presents a consolidated technology scenario, with a large number of patent documents and a low number of scientific papers. Brazil is in a scenario of potential application, but it is in a phase of technological development, but the initiatives of the universities stand out, developing technologies aimed at this market and making the deposit, giving them visibility within the sector industrial.
\end{abstract}

Keywords: Food Engineering. Technological foresight. Malting.

Área tecnológica: Prospecção tecnológica. 


\section{Introdução}

O setor industrial, não raramente, faz uso dos avanços tecnológicos, resultado de pesquisas científicas, realizadas nas mais diversas áreas da ciência, como a química, biologia, farmácia ou engenharia, com o objetivo final de atender às demandas do mercado consumidor (SILVA, 2017). Nesse contexto, as inovações tecnológicas são assimiladas pelas indústrias e disponibilizadas na forma de bens de consumo ou como serviços à sociedade, ou atuam no aprimoramento das várias fases da cadeia produtiva. Essa interação, entre os meios industrial e científico, provoca mudanças na sociedade como um todo e, também, no modo como os seus integrantes se relacionam (EARLE, 1997). O mesmo autor discorre sobre a influência das atividades inovadoras na indústria de alimentos, detalhando suas características e, principalmente, seus efeitos sociais e culturais. Ainda, finaliza seu estudo com uma reflexão sobre a importância que a indústria de alimentos deve dedicar às consequências sociais e ambientais de suas estratégias de inovação, a fim de bem atender os desejos do mercado consumidor, considerando não apenas os aspectos nutricionais, mas também pessoais e sociais.

Diversos outros estudos abordam a inovação na indústria alimentar, podendo ser citado Martinez (2000), que realiza uma análise da característica evolutiva, em vez de revolucionária, das atividades inovadoras na indústria de alimentos e bebidas espanhola. Segundo Martinez (2000) além da resistência, por parte dos comerciantes, em aceitarem vender novos produtos, destaca-se a importância do desenvolvimento nacional de estratégias de inovação para o setor industrial, fazendo uma correlação direta entre o sucesso na inserção no mercado de novos produtos e a autonomia tecnológica da indústria nacional.

Bigliardi e Galati (2013) apresentam a importância da indústria de alimentos, para a economia da Itália e União Européia como um todo, vista como o principal ator na manufatura das matérias-primas agrícolas e no fornecimento de alimentos. O estudo destaca ainda a forte presença de tecnologias inovadoras nesse setor. Ela usa as comidas funcionais como exemplo de pesquisa na indústria alimentícia. Esse tipo de alimento oferece, além do aporte nutricional básico, uma promoção na condição de saúde dos consumidores, devido à adição de suplementos, como o suco de laranja com adição de cálcio.

No entanto, a influência e o potencial das tecnologias inovadoras na indústria já eram tópicos de discussão fora do meio acadêmico muito antes dos trabalhos de Martinez (2000) e de Bigliardi e Galati (2013), como explica Martin, em seu artigo de 1995. Nesse trabalho, são comparados os casos dos governos do Japão, Estados Unidos, Holanda, Alemanha, Austrália, Nova Zelândia e Reino Unido, ao fazerem uso de técnicas de prospecção como ferramentas de alocação de recursos, a fim de identificar as tecnologias emergentes inovadoras mais promissoras e que possuíssem o maior potencial de retorno positivo para economia e sociedade (MARTIN, 1995).

Nesse contexto de utilização de técnicas de prospecção para detectar tecnologias inovadoras $e$, consequentemente, o seu potencial de apropriação pela indústria torna-se uma ferramenta relevante ligada ao desenvolvimento de produtos e processos inovadores, junto à indústria. O presente estudo tem o objetivo de apresentar o panorama internacional e nacional de processos e equipamentos, utilizados na produção do malte, a fim de permitir uma avaliação do potencial 
de mercado de tecnologias desenvolvidas nesse setor tecnológico da indústria de alimentos $e$ bebidas. Sendo assim, o estudo, apresentado no presente artigo, aplicou uma metodologia de prospecção para investigar o panorama internacional e nacional, na indústria de alimentos $e$ bebidas, mais especificamente o setor cervejeiro, de tecnologias e processos de produção de malte, utilizado como matéria-prima para a fabricação de bebidas alcoólicas, analisando a participação dos principais países, companhias e Institutos de Ciência e Tecnologia (ICTs) atuantes nesse setor.

Diversos exemplos de aplicação das técnicas de prospecção tecnológica na indústria de alimentos podem ser encontrados na literatura, por exemplo,, as análises a respeito de tecnologias relacionadas ao queijo de coalho (MENESES, 2011), às aplicações da planta de babaçu nos segmentos de cosméticos e de gêneros alimentícios (PINHEIRO, 2015), à fabricação de alimentos em barras de cereais, empregando resíduos (UCHÔA, 2015) e à produção de produtos à base de quinoa (PANZOLINI, 2017).

A importância deste estudo consiste em apresentar o estado dos avanços tecnológicos e do mercado de produção de malte, levando em consideração que o Brasil é uma potência agroindustrial, sendo o terceiro maior produtor mundial de cerveja, com uma produção anual de 14 bilhões de litros de cerveja, ficando atrás da China, primeiro lugar, e dos Estados Unidos, segundo lugar (BARTH-HAAS GROUP, 2015). O cenário mundial e nacional do mercado cervejeiro mostra que esse setor se caracteriza pelo grande potencial para o crescimento em número depósito de proteções de equipamentos, utilizados na produção da cerveja. Nas duas últimas décadas, observou-se a hegemonia do setor industrial nos depósitos de pedidos de patente nessa área, cerca de 97\%, com destaque para a empresa japonesa Asahi Breweries, responsável por 18,8\% dos depósitos (FERNANDES et al., 2017).

Outro fator a se considerar é a grande influência da interação entre as ICTs e o setor industrial e o mercado, discutido em Lima et al. (2017). Em nível nacional, o desenvolvimento da indústria brasileira de bebidas, em especial, do setor cervejeiro na região Centro-Oeste, apresentou um grande avanço na última década e se consolidou como um dos setores mais dinâmicos da economia brasileira, especialmente no que diz respeito à criação e à expansão de novos nichos comerciais (LIMA et al., 2017).

\section{Metodologia}

A metodologia do presente estudo faz uso de técnicas de prospecção tecnológica para avaliar, em um primeiro momento, a partir do panorama internacional científico e tecnológico, o potencial de mercado de tecnologias para produção de malte a base de grãos; em um segundo momento, é analisado o panorama nacional das proteções patentárias e o papel desempenhado pelas ICTs nesse setor tecnológico.

Com essa metodologia, foram analisados dois tipos de documentos: documentação patentária e artigos científicos. A documentação patentária foi utilizada como fonte de indicadores para medir o nível de domínio da tecnologia, enquanto que os artigos científicos forneceram indicadores sobre o nível de conhecimento científico no campo estudado. 
Os documentos analisados foram obtidos por meio de pesquisas realizadas na plataforma de busca e análise de documentação patentária Orbit (QUESTEL, 2018), que consolida documentos publicados nas bases do Instituto Nacional da Propriedade Industrial (INPI), do Organização Mundial de Propriedade Intelectual (OMPI), do Escritório de Patentes e Marcas dos Estados Unidos (USPTO) e do Escritório de Patentes Europeu (EPO), na base de publicações científicas Web of Science (CLARIVATE ANALYTICS, 2018).

As pesquisas, nas plataformas Orbit (QUESTEL, 2018) e Web of Science (CLARIVATE ANALYTICS, 2018), foram realizadas utilizando combinações de palavras-chave, a fim de melhor representar o objeto de pesquisa, inseridas nos campos "título" ou "resumo". Foram utilizadas as seguintes palavras-chave: malt, malting, brewing, process, production, cereal e grain. A delimitação dos resultados à área desejada foi feita por meio da aplicação de um filtro para restringir a busca aos domínios técnicos Chemical Engineering, Biotechnology, Food Chemistry, para a plataforma Orbit (QUESTEL, 2018), e Food Technology, Chemical Engineering e Biotechnlogy Applied to Microbiology para a plataforma Web of Science (CLARIVATE ANALYTICS, 2018). Em ambas as bases foi definido um intervalo de pesquisa de 10 anos, de 01/01/2008 até 31/12/2017. As combinações de palavras-chave foram construídas utilizando técnicas clássicas de busca, como truncamento de palavras (*, \$, ?) e operadores booleanos (AND, OR e NOT).

Os resultados obtidos foram analisados, a fim de identificar a evolução anual do número de documentos patentários depositados e de artigos científicos publicados, os principais países de prioridade, as empresas que possuem maior número de depósitos, a contribuição dos principais países de prioridade na evolução anual dos documentos patentários, as principais Classificações Internacionais de Patentes (IPC) das tecnologias depositadas, a situação legal das proteções e o grau de domínio da tecnologia por país.

\section{Resultados e Discussão}

Nesta seção serão apresentados os resultados, iniciando-se pelo panorama internacional, seguido do cenário brasileiro.

\subsection{Pesquisa de Documentos Patentários}

A estratégia de busca, utilizada na plataforma Orbit (QUESTEL, 2018), para uma data de prioridade situada entre 01/01/2008 até 31/12/2017, é apresentada na Tabela 1. Foram testadas três combinações de palavras-chave, diferenciadas entre si pelos seus graus de complexidade crescente, em razão do acréscimo de sinônimos para as palavras-chave, combinado com técnicas de truncamento e uso de operadores booleanos. Observou-se um aumento no número de resultados, conforme a complexidade da combinação de palavras-chave foi aumentada, obtendo-se para a terceira combinação (malt or malting or brew*) and (process* or product*) and (cereal* or grain*) um total de 2.109 documentos. Tendo em vista que o presente trabalho utiliza-se de técnicas de prospecção tecnológica, a fim de definir o panorama global de tecnologias nas áreas de biologia, química e engenharia de alimentos, voltadas para produção de malte, optou-se 
por adotar um universo de documentos mais abrangente. Essa opção foi feita com o intuito de representar, da maneira mais adequada possível, o objeto do presente estudo e fornecer uma quantidade expressiva de documentos, possibilitando, assim, uma análise clara e representativa do panorama internacional das documentações patentárias relacionadas à tecnologia analisada.

Tabela 1 - Resultados da busca de anterioridade, por combinações de palavras-chave, de 2008 a 2017, com filtro por domínio técnico Chemical Engineering, Biotechnology, Food Chemistry

\begin{tabular}{|c|c|c|}
\hline ÍNDICE & COMBINAÇÕES DE PALAVRAS-CHAVE & N. DE DOCUMENTOS \\
\hline 1 & (malt or malting or brew*) and (process $\left.{ }^{*}\right)$ and (cereal*) & 405 \\
\hline 2 & (malt or malting or brew*) and (process* or product*) and cereal* & 539 \\
\hline 3 & (malt or malting or brew*) and (process* or product*) and (cereal* or grain*) & 2109 \\
\hline
\end{tabular}

Fonte: Elaborada pelos autores deste artigo (2018)

O resultado final da busca, 2.109 documentos patentários, foi utilizado para analisar o comportamento das proteções das tecnologias para produção de malte, a partir de grãos e cereais, por meio da análise dos principais países de prioridade, da evolução anual do número de pedidos depositados, considerando a contribuição dos principais países de prioridade, dos principais depositantes, dos principais códigos IPC's depositados e da situação legal dos documentos.

Na Figura 1, na qual é apresentada a distribuição dos documentos patentários, depositados de 2008 a 2017, com relação aos 10 principais países de prioridade, pode-se observar que 1.436 documentos, $68 \%$ dos documentos depositados, apresentaram a China como país de prioridade, seguida pela Rússia com 401 documentos (19\%), sendo uma parcela de $13 \%$ dos demais documentos distribuída entre os demais países de prioridade, incluindo aqueles que não se encontram entre os 10 principais países de prioridade. Segundo Hu e Jefferson (2009), o grande número de depósitos realizados na China deve-se ao aumento dos investimentos em atividades de pesquisa e desenvolvimento, acompanhado do crescimento dos investimentos estrangeiros na economia chinesa, o que incentiva as empresas chinesas a depositarem mais pedidos de patente. Em paralelo a esses elementos, também devem ser consideradas as emendas à legislação que favorecem titulares de patentes e a reforma no direito de propriedade que esclareceu a atribuição de direitos de propriedade.

Analisando de maneira mais aprofundada a participação da China (68\%) e da Rússia (19\%), como apresentado na Figura 2, durante o período de prioridade que vai de 2008 a 2017, nota-se que a curva do número de depósito de documentos da China (linha azul) apresenta um comportamento de crescimento contínuo, de 2008 até 2016, com um aumento expressivo do número de proteções em 2011, e um declínio em 2017. Em relação à Rússia (linha vermelha), a mesma análise apresenta uma curva constante, de 2008 até 2014 e de 2016 até 2017. No ano de 2015, a Rússia apresentou 305 depósitos, o que corresponde a mais de 50\% das proteções em comparação com os outros anos, ultrapassando o indicador de depósitos da China (234 documentos). 
Figura 1 - Distribuição dos documentos patentários, depositados no período de 2008 a 2017, entre os 10 principais países de prioridade

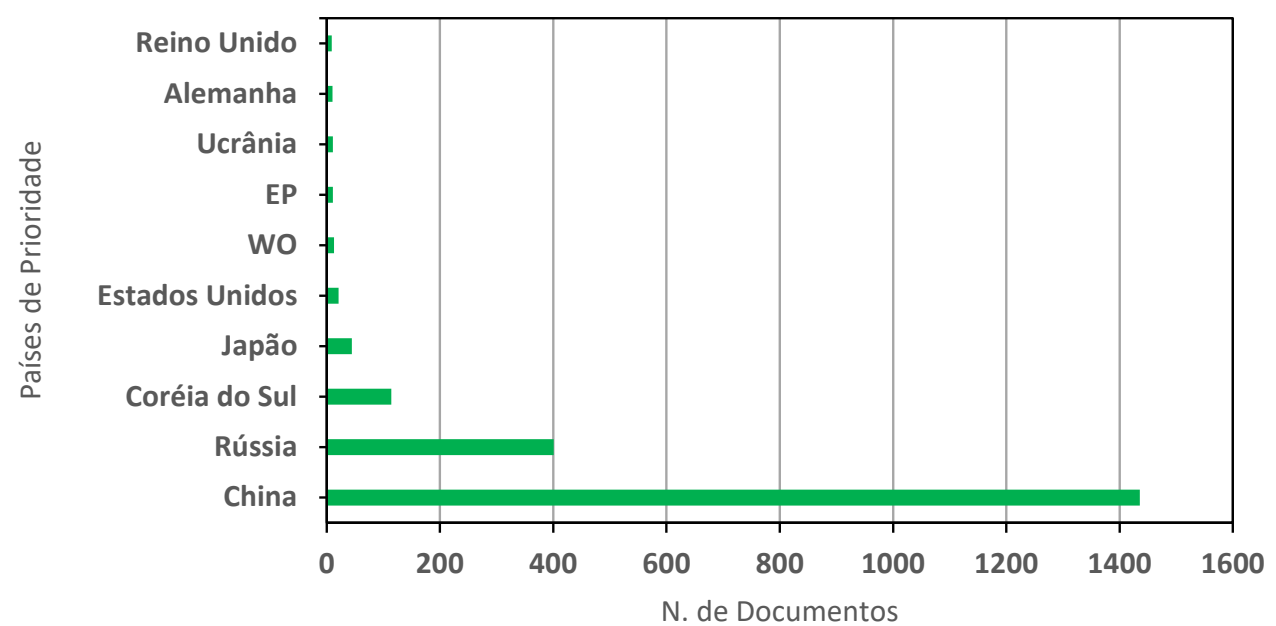

Fonte: Elaborada pelos autores deste artigo (2018)

A análise da distribuição dos documentos patentários entre os 10 principais depositantes, de 2008 a 2017 (Figura 3) apresenta uma participação exclusiva de indústrias, centros de pesquisa e de universidades chinesas. Esse resultado corrobora com a distribuição do número de depósitos por países de prioridade (Figura 1). Entre os 10 primeiros depositantes, observa-se a presença de oito indústrias e de duas universidades, sendo que a universidade melhor posicionada (Jiangnan University) encontra-se em terceiro lugar na lista.

A partir da análise da classificação por área de conhecimento, apresentada pelos resultados da busca, observou-se que mais de 600 documentos, ou seja, $32 \%$, estão concentrados na classificação C12G, que corresponde à classe "Química, Metalurgia", subclasse "Química, Vinho", mais especificamente à preparação de bebidas alcoólicas; e aproximadamente 350 documentos, $17 \%$, apresentam a classificação A23L, referente à classe "Necessidades Humana", subclasse "Alimentos, produtos alimentícios ou bebidas não alcoólicas".

Figura 2 - Apresentação da contribuição anual da China (linha azul) e da Rússia (linha vermelha) na evolução anual dos documentos patentários, com data de prioridade de 2007 a 2017

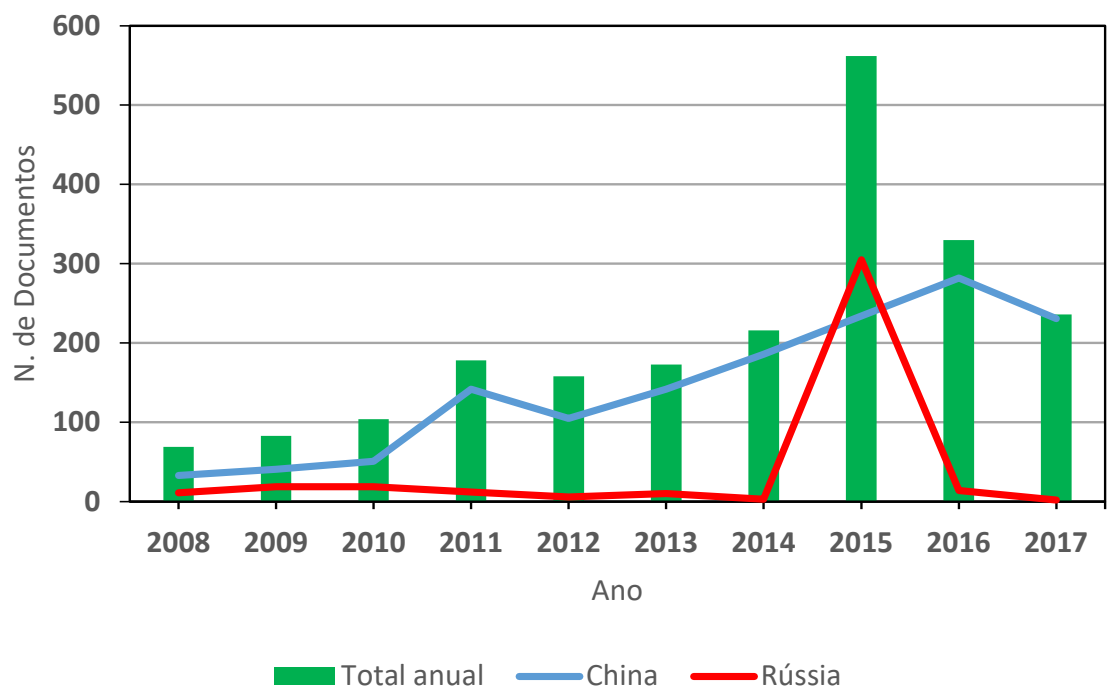

Fonte: Elaborada pelos autores deste artigo (2018) 
Figura 3 - Distribuição dos documentos patentários entre os 10 principais depositantes, no período de 2008 a 2017

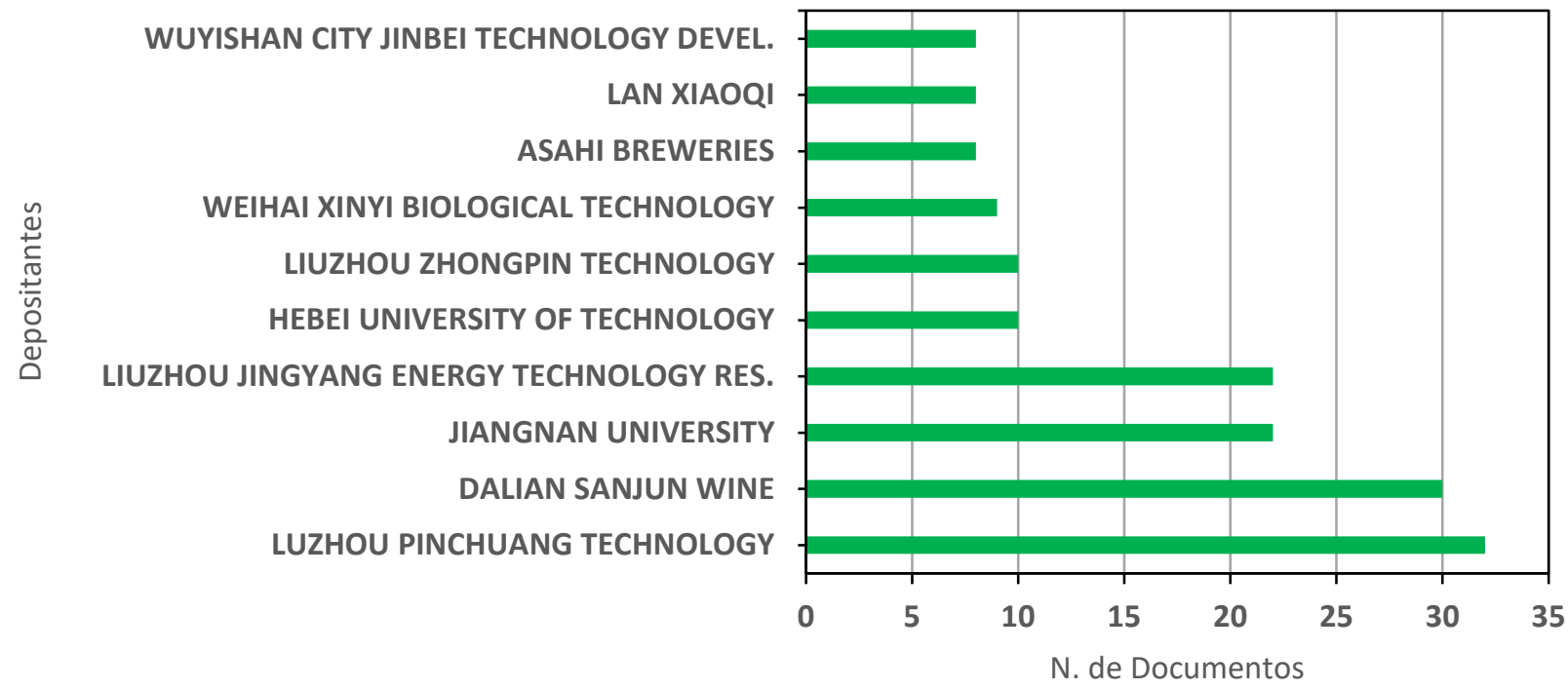

Fonte: Elaborada pelos autores deste artigo (2018)

A situação legal dos documentos patentários também foi analisada. Os documentos encontrados foram divididos em quatro categorias: expirado, revogado, em análise e concedido. Foi observado que mais de 800 documentos, cerca de $38 \%$, apresentam o status de concedido, e que cerca de 780 documentos, $37 \%$, ainda estão em avaliação. Os pedidos de patentes expirados ou revogados somam 508 documentos (24\%).

\subsection{Pesquisa de Artigos Científicos}

A pesquisa na base de artigos científicos Web of Science (CLARIVATE ANALYTICS, 2018), para estudos de tecnologias para produção de malte a partir de grãos ou cereais, nas áreas de Tecnologia de Alimentos, Engenharia Química, Biotecnologia Aplicada à Microbiologia, apresentou como resultado um total de 723 documentos (Tabela 2).

Tabela 2 - Resultados da pesquisa em base de artigos científicos, por combinação de palavras-chave, publicados de 2008 a 2017, utilizando o filtro por setor Food Technology, Chemical Engineering e Biotechnlogy Applied to Microbiology

\begin{tabular}{cc} 
Combinação de PALAVRAS-CHAVE & No DE ARTIGOS \\
(malt or malting or brew*) and (process* or product*) and (cereal* or grain*) & 723 \\
\hline
\end{tabular}

Fonte: Elaborada pelos autores deste artigo (2018)

O resultado de artigos científicos foi utilizado para avaliar o panorama das pesquisas, voltadas para a tecnologia objeto do presente estudo. Primeiramente, verificou-se a distribuição anual de artigos publicados, de 2008 a 2017 (Figura 4). Nessa análise, observou-se um comportamento na curva do número de publicações, 60 artigos/ano, de 2008 a 2012. De 2013 a 2017, observou-se um aumento no número de publicações, atingindo um valor máximo de 105 artigos em 2015. 
Figura 4 - Distribuição anual da publicação artigos científicos, de 2008 a 2017

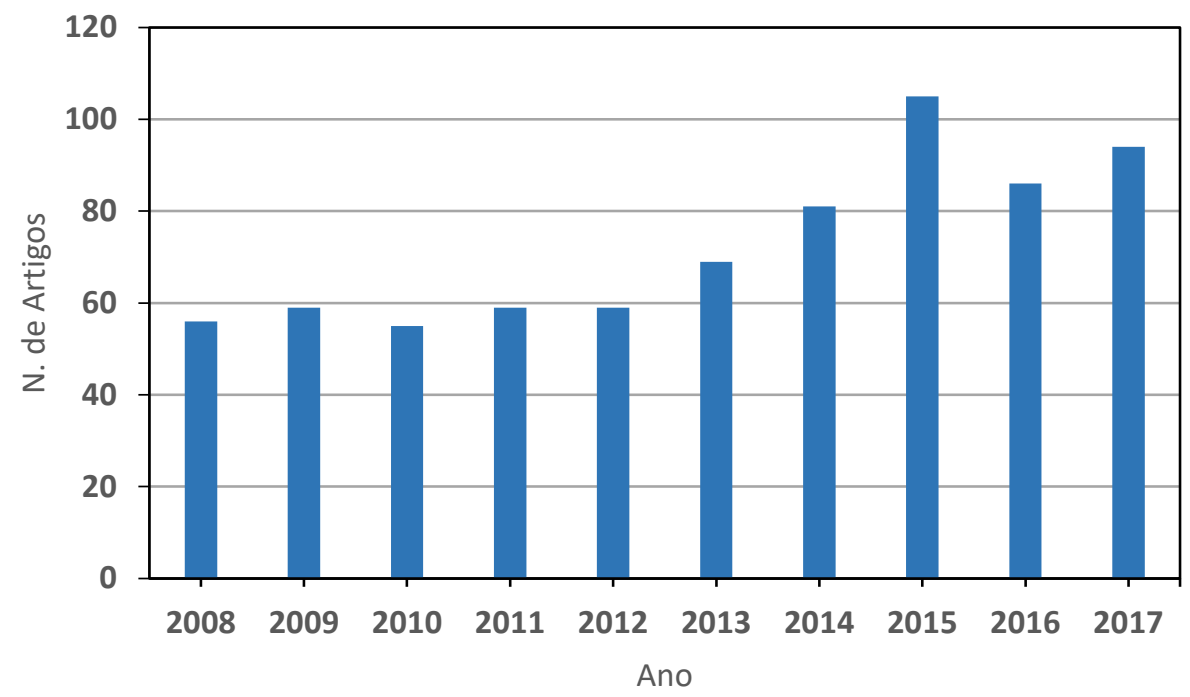

Fonte: Elaborada pelos autores deste artigo (2018)

Analisando esses resultados em relação aos 10 principais países de publicação (Figura 5), que concentram 63\% das publicações nessa área, observou-se que a Alemanha é a líder em publicações com 69 artigos, seguida da Irlanda com 53. O Brasil e a China encontram-se em terceiro lugar em termos de artigos publicados, com 48 artigos cada.

Figura 5 - Distribuição do número artigos publicados entre os 10 primeiros países, de 2008 a 2017

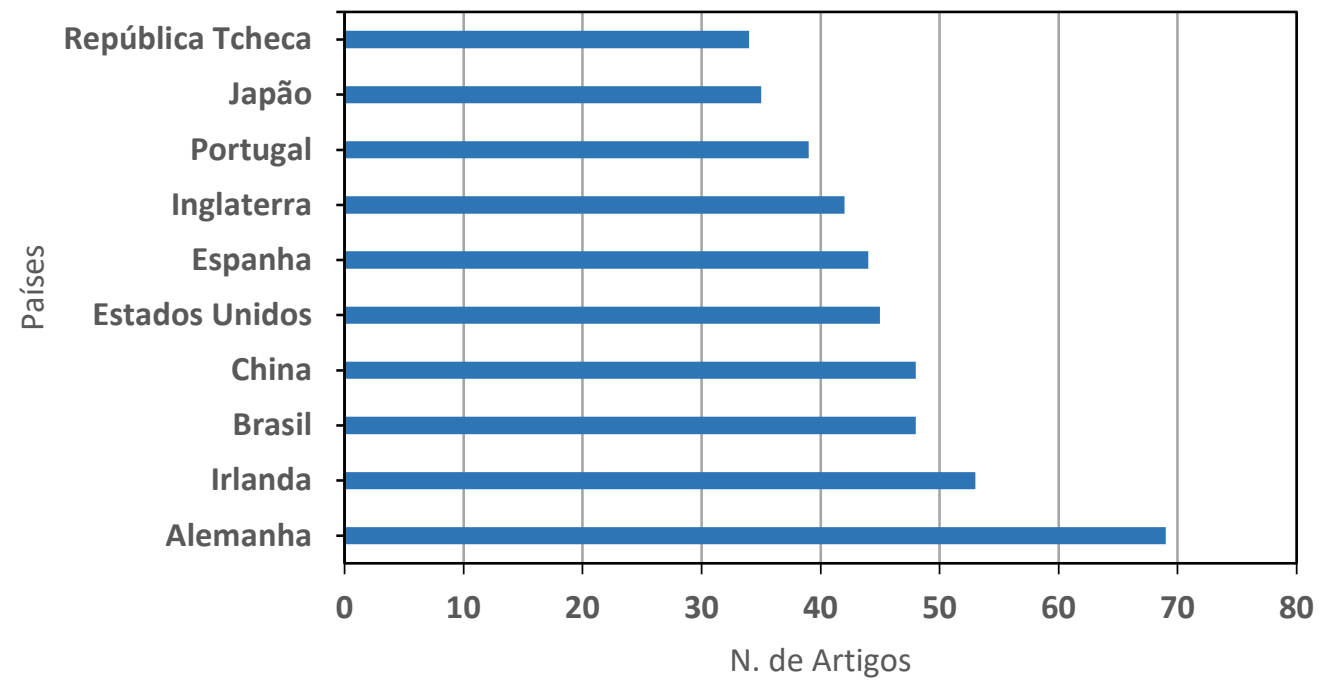

Fonte: Elaborada pelos autores deste artigo (2018)

A dispersão anual da relação entre artigos e documentos patentários, de 2008 a 2017, é apresentada na Figura 6. Essa análise permite identificar a contribuição individual da produção científica, representada pelos artigos científicos (porção azul das colunas) e da documentação patentária (porção verde das colunas) no total da produção científica e tecnológica de cada ano. Uma análise semelhante a essa foi aplicada por Quintella et al. (2009), em seu trabalho de prospecção tecnológica aplicada à área de biodiesel. Na análise do presente estudo, observa-se que, durante todo o período analisado, a participação das documentações patentárias foi superior a apresentada pelos artigos científicos, em todos os anos. Os documentos patentários 
apresentaram uma participação mínima de 55\% (69 registros), em 2008, e uma máxima de 84\% (562 documentos), em 2015.

Esse comportamento é típico de tecnologias que despertam grande interesse por parte do setor industrial, ainda mais por se tratarem de tecnologias empregadas na produção de insumos de bebidas alcoólicas, um forte ramo do setor de alimentos, que, segundo dados da empresa de consultoria Allied Market Research (KUMAR, 2017), o mercado mundial de bebidas alcoólicas atingiu um valor US \$ 1,344 bilhões em 2015, e estima-se que chegará a US $\$ 1,594$ bilhões em 2022. No Brasil, esse mercado faturou $\mathrm{R} \$ 107$ bilhões em 2007, e que corresponde a 1,6\% do PIB, segundo dados da Associação Brasileira da Indústria da Cerveja (CERVBRASIL, 2018). Ainda segundo relatório do BNDES (CERVIERI JÚNIOR et al., 2014), o Brasil é o terceiro mercado mundial, atrás da China (primeira) e dos EUA (segundo). Pode-se atribuir a esses dados o constante interesse do meio acadêmico no desenvolvimento de tecnologias e processo nessa área, como pode ser verificado pelos artigos científicos apresentarem uma contribuição que variou de uma mínima de 16\% (105 registros), em 2015, a uma máxima de 45\% (56 registros), em 2008.

Figura 6 - Dispersão anual da relação entre artigos e documentos patentários, de 2007 a 2017

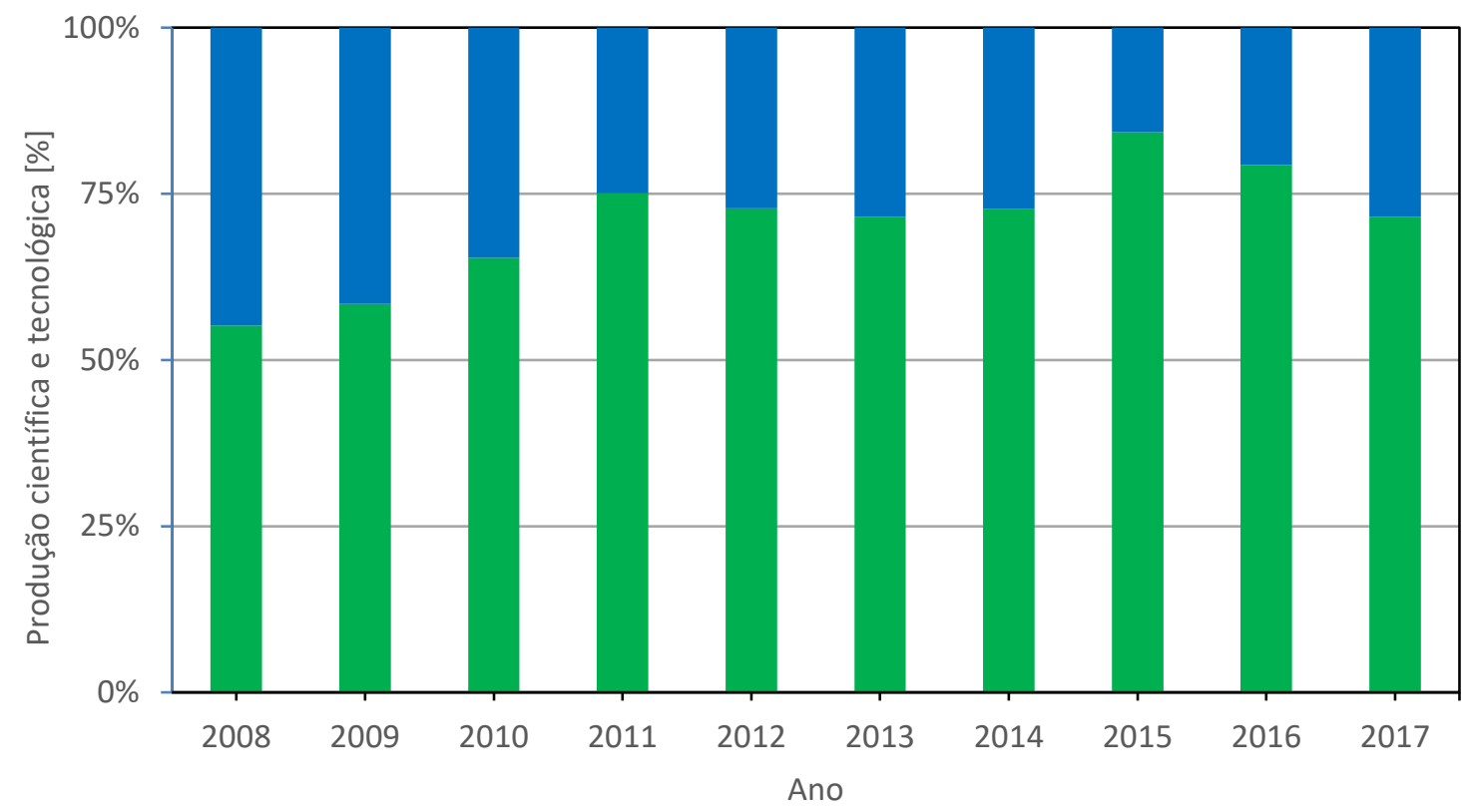

- Documentação patentária

Artigos científicos

Fonte: Elaborada pelos autores deste artigo (2018)

Na Figura 7, os 10 principais países de prioridade e de publicação de artigos foram comparados entre si. Nessa análise, o domínio tecnológico (patentes), no eixo horizontal, e o domínio científico (artigos), no eixo vertical, são usados para posicionar cada um dos países, de acordo com as suas contribuições em cada área. O tamanho dos círculos representa a fração entre o número de documentos patentários dos países em relação à produção total, que corresponde à soma de artigos e de patentes, de 2008 a 2017. A partir dessa análise, pode-se observar que apesar da China possuir um número médio de artigos publicados (48 artigos), ela encontra-se isolada no lado direto do gráfico, devido à sua hegemonia em documentos patentários (1.436 patentes). Países desenvolvidos como Japão (44 patentes), Estados Unidos (21 patentes), Alemanha (10 patentes), Reino Unido ( 9 patentes) e Austrália ( 4 patentes) encontram-se posicionados 
sobre o eixo vertical, do lado esquerdo do gráfico, devido ao número de documentos patentários nos quais eles aparecem como países de prioridade ser muito inferior aos da China. A Rússia (401 patentes) e a Coréia (114 patentes) aparecem em uma posição intermediária, devido aos seus indicadores para patentes, mas sobre o eixo horizontal, devido à baixa produção científica nessa área.

Em relação à participação de cada país na produção científica total, mensurada pelo tamanho do círculo, verifica-se um isolamento da China, seguida da Rússia e da Coréia, demonstrando uma assimilação das tecnologias por parte das indústrias chinesas, enquanto que as russas $e$ coreanas encontram-se em fase de apropriação. Observa-se, ainda, que os Estados Unidos, a Alemanha e o Reino Unido estão próximos em termos de documentos patentários protegidos, mas em proporção muito inferior àquela apresentada pela China, Rússia e Coréia. Esta análise fornece informações complementares à análise de dispersão da relação entre artigos e patentes, Figura 6, uma vez que aquela fornece um panorama global do nível de transferência da tecnologia do meio científico para o industrial, enquanto que a dispersão da relação entre patentes e produção científica e tecnológica permite uma avaliação da situação de cada país, de 2008 a 2017.

Figura 7 - Dispersão da relação entre patentes e produção científica e tecnológica por país, de 2008 a 2017

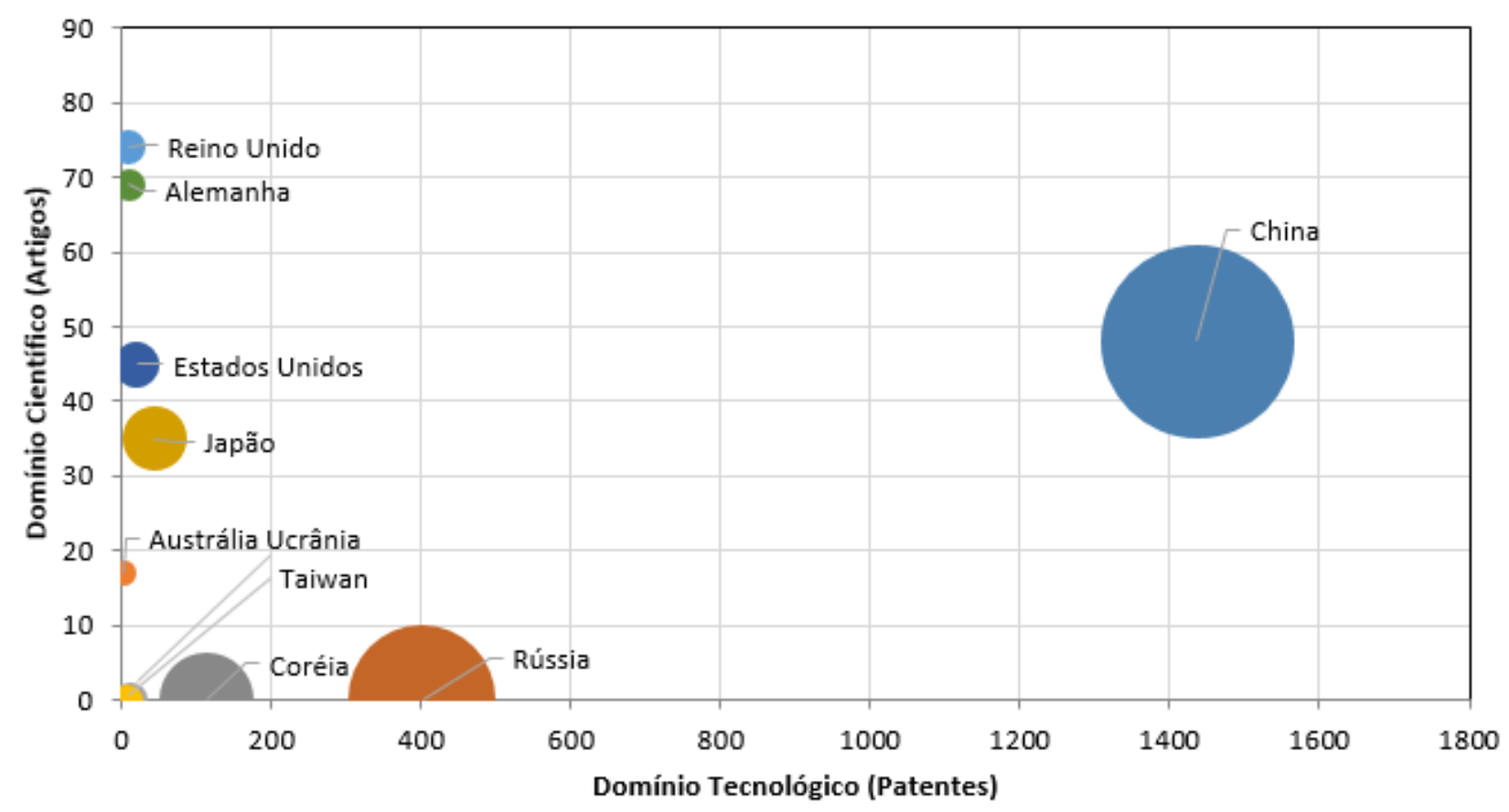

Fonte: Elaborada pelos autores deste artigo (2018)

\subsection{Panorama Nacional}

Os resultados obtidos pela pesquisa na plataforma Orbit (QUESTEL, 2018) não forneceram nenhum registro referente ao Brasil como um país de prioridade. No entanto, a fim de verificar qual o panorama nacional de proteção de tecnologias para produção de malte, foi realizada uma pesquisa na base de patentes do INPI (INPI, 2018), com data de prioridade de 01/01/2008 
e 31/12/2017, a inserindo a combinação de palavras-chave, (malte or malteação or ferment*) and (process* or produç*) and (grão* or cerea*), apenas no campo resumo.

Essa pesquisa apresentou um total de 16 documentos patentários. A dispersão anual desses documentos, de 2008 a 2017 (Figura 8), mostra que os depósitos ficaram concentrados de 2010 a 2014, com um único registro em 2016. Não foram retornados registros de 2008 e de 2009, e em 2015. Esse resultado mostra apenas que não foram realizados depósitos nesse período com data de prioridade nesses anos, no entanto, é possível que existam registros de depósitos para anos de prioridade anteriores a 2008.

Dentre estes 16 resultados, apenas um registro é de titularidade de um Instituto de Ciência e Tecnologia australiano, os demais registros, 93\%, foram realizados por indústrias, em sua maioria por empresas dos Estados Unidos. Uma análise da dispersão dos documentos por países de prioridade (Figura 9) mostra que os $62,5 \%$ dos depósitos apresentam os Estados Unidos como país de prioridade. O Brasil não aparece como país de prioridade, durante o período analisado. Analisando esses indicadores e a produção científica brasileira, nesse mesmo período, tem-se 48 artigos e pode-se concluir que, em nível nacional, as tecnologias de produção de malte ainda não começaram sua transferência para o setor industrial.

Figura 8 - Dispersão anual da documentação patentária, de 2008 a 2017, pesquisada na base de patentes do INPI

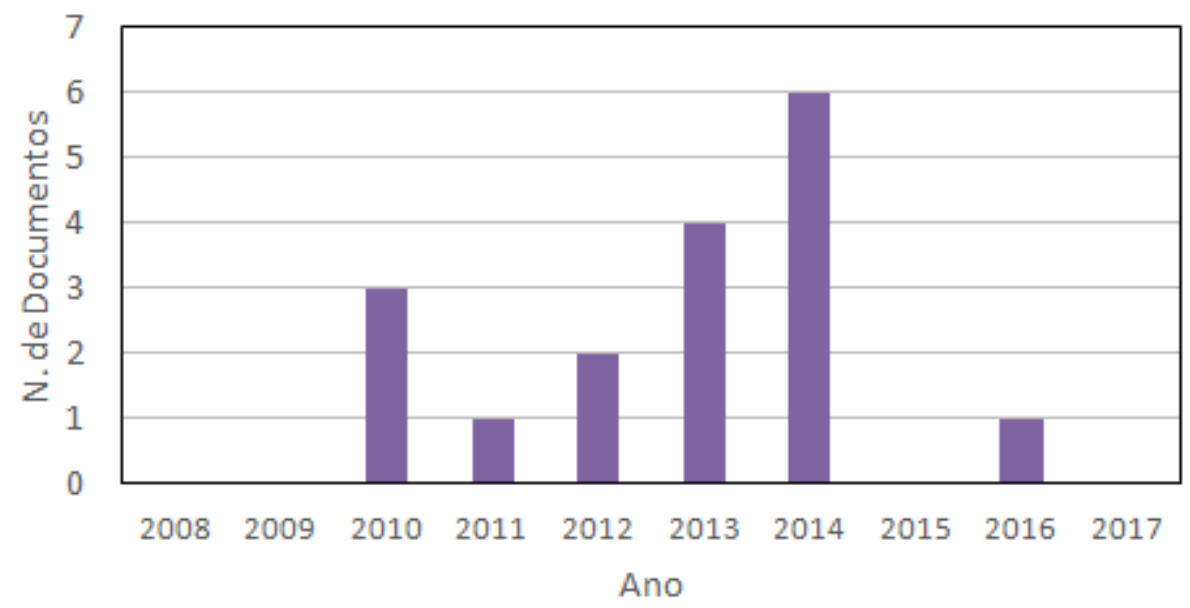

Documentos Patentários

Fonte: Elaborada pelos autores deste artigo (2018)

Esse comportamento é característico de tecnologias em fase de pesquisa científica, que ainda não apresentam uma maturidade tecnológica que permita a sua transferência para o setor industrial. Como exemplo, pode-se citar o pedido de patente BR 102018 011822-6 (GHESTI et al., 2018), depositado, junto ao INPI, em junho de 2018, pela Fundação Universidade de Brasília (FUB), que está sobre o prazo de sigilo de 18 meses. Essa tecnologia trata de um processo e equipamento para produção de malte. A mesma teve o seu nível de maturidade avaliado, seguindo o método descrito em Mankins (2009), e recebeu uma classificação TRL 4, pois foi testada apenas em escala laboratorial. 
Figura 9 - Distribuição dos resultados da pesquisa no INPI, por país de prioridade

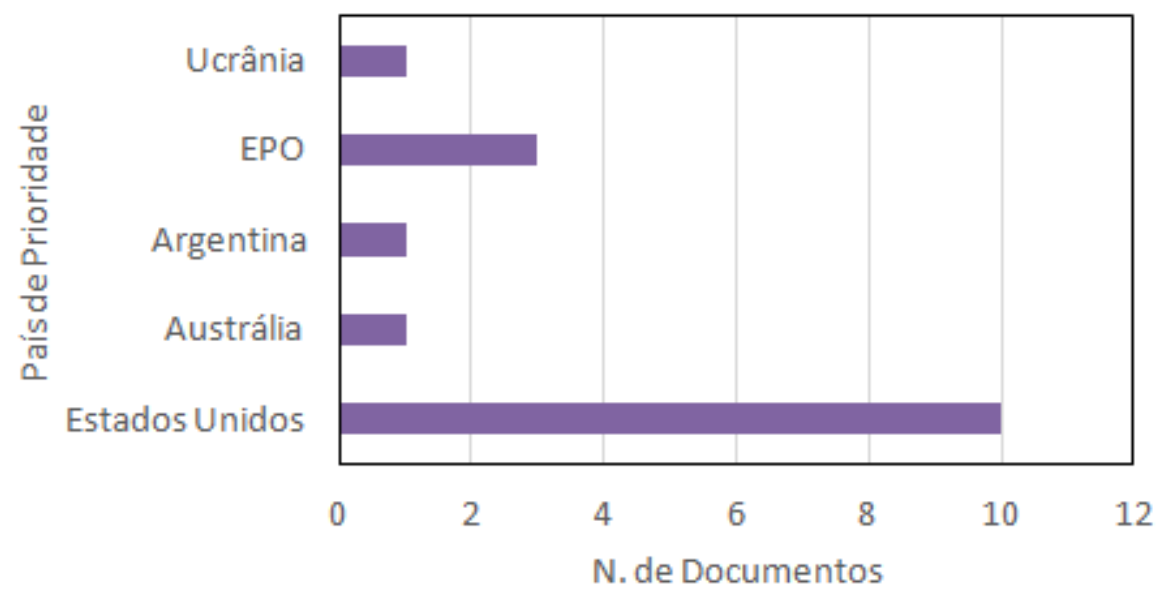

Fonte: Elaborada pelos autores deste artigo (2018)

O depósito de pedido de patente BR 102018 011822-6 é um exemplo das tecnologias desenvolvidas dentro dos ICTs. Em sua grande maioria, elas apresentam TRL inferiores a 5, e para alcançarem níveis mais elevados de maturidade, necessitam de desenvolvimentos que exigem estruturas físicas, recursos humanos e aportes financeiros não acessíveis às universidades.

\section{Considerações Finais}

Em uma avaliação por país, nota-se que a China destaca-se em produção de documentos patentários, seguida pela Rússia e pela Coréia, e que os demais países ainda encontram-se em fase inicial de transferência da tecnologia. A China apresenta um cenário de tecnologia consolidada, com um grande número de documentos patentários e baixo número de artigos científicos.

Do ponto de vista nacional, os documentos patentários depositados no INPI, obtidos por meio de pesquisas na base de patentes do INPI, de 2008 a 2017, são todos de prioridade estrangeira. Destacam-se entre os países de prioridade os Estados Unidos com 10 dos 16 documentos encontrados. Dos 16 documentos patentários, apenas um possui como titular uma instituição de pesquisa australiana, o que indica que, no Brasil, esse tipo de tecnologia ainda encontra-se em fase de desenvolvimento dentro dos laboratórios de pesquisa, e que ainda não se iniciou a sua apropriação pelo setor industrial brasileiro.

Um exemplo desse cenário é a tecnologia para produção de malte, desenvolvida na FUB, pedido de patente BR 102018 011822-6 (GHESTI et al., 2018), depositado no INPI em junho de 2018, que por possuir um nível de TRL igual a 4 não apresenta um grau de maturidade tecnológico necessário para sua incorporação pela indústria, mas se destaca seu papel pioneiro e de vanguarda ao proteger uma tecnologia em um setor de grande impacto financeiro, como a indústria de alimentos e bebidas. Nesse contexto, apesar de o Brasil encontrar-se em um cenário no qual as tecnologias ainda não atingiram um nível de maturidade suficiente para serem assimiladas pela indústria, destacam-se as iniciativas das universidades em tentar mudar esse quadro, ao desenvolverem tecnologias voltadas para esse mercado e realizarem o seus depósito, conferindo-lhes visibilidade dentro do setor industrial. 
Diante do cenário agroindustrial do país, existe um ambiente promissor para o desenvolvimento de tecnologias que agreguem valor a cereais cultivados, uma vez que o Brasil é um dos maiores exportadores de grãos. Ainda, a necessidade de importação de malte de cevada para a indústria cervejeira, demonstra a preocupação e a necessidade do setor em adquirir matéria-prima produzida nacionalmente. Sendo assim, nos próximos anos a agroindústria e a indústria alimentícia necessitarão incorporadas novas tecnologias relacionadas à manufatura de cereais.

Por fim, tomando-se como exemplo o desempenho chinês, pode-se observar que a inovação no setor industrial necessita de um ambiente propício para se desenvolver, no qual os incentivos financeiros promovem o crescimento das empresas e o arcabouço legal protege o direito à propriedade industrial. Conclui-se, portanto, que o panorama nacional poderá ser alterado, nas próximas décadas, se medidas econômicas e políticas, que incentivem o parque industrial, forem adotadas em conjunto com ações legislativas que favoreçam a disseminação da propriedade industrial.

\section{Referências}

ASSOCIAÇÃO BRASILEIRA DA INDÚSTRIA DA CERVEJA (CERVBRASIL). Dados do Setor Cervejeiro Nacional. 2018. Disponível em: <http://www.cervbrasil.org.br/novo_site/dados-dosetor/>. Acesso em: jul. 2018.

BARTH-HAAS GROUP. The Barth Report HOPS 2014/2015. 2015. Disponível em: < https:// www.barthhaasgroup.com/images/mediacenter/downloads/pdfs/412/barthreport2014-2015en.pdf>. Acesso em: ago. 2018.

BIGLIARDI, Barbara; GALATI, Francesco. Innovation trends in the food industry: The case of functional foods. Trends in Food Science \& Technology, [S.1.], v. 31, n. 2, p. 118-129, 2013. Disponível em: <https://www.sciencedirect.com/science/article/abs/pii/S0924224413000678>. Acesso em: 28 jun. 2018.

CERVIERI JÚNIOR, Osmar et al. O setor de bebidas no Brasil. BNDES Setorial, Rio de Janeiro, n. 40, p. 93-129, set. 2014. Disponível em: < https://web.bndes.gov.br/bib/jspui/bitstream/1408/3462/1/ BS\%2040\%200\%20setor\%20de\%20bebidas\%20no\%20Brasil_P.pdf > . Acesso em: 28 jun. 2018.

CLARIVATE ANALYTICS. Web of Science. 2018. Disponível em: < http://login.webofknowledge. com>. Acesso em: 28 jun. 2018.

EARLE, M. D. Innovation in the food industry. Trends in Food Science \& Technology, [S.1.], v. 8, n. 5, p. 166-175, 1997. Disponível em: < https://www.sciencedirect.com/science/article/abs/pii/ S0924224497010261>. Acesso em: 28 jun. 2018.

FERNANDES, Thiago Lara et al. Prospecção tecnológica: uma visão das inovações e perspectivas do mercado cervejeiro. Cadernos de Prospecção, Salvador, v. 10, n. 4, p. 851-865, 2017. Disponível em: <https:/portalseer.ufba.br/index.php/nit/article/view/23039>. Acesso em: ago 2018.

GHESTI, Grace Ferreira et al. Reator de câmara única e processo para malteação de grãos em leito fluidizado. Brasília, DF, BR 102018011822 6, 2018. Disponível em: <https://gru.inpi.gov.br/ pePI/servlet/PatenteServletController>. Acesso em: 28 jun. 2018.

HU, Albert Guangzhou; JEFFERSON, Gary H. A great wall of patents: What is behind China's recent patent explosion? Journal of Development Economics, [S.1.], v. 90, p. 57-68, 2009. Disponível 
em: <https://www.sciencedirect.com/science/article/pii/S0304387808001120>. Acesso em: 28 jun. 2018.

\section{INSTITUTO NACIONAL DA PROPRIEDADE INDUSTRIAL (INPI). PEPI - Pesquisa em}

Propriedade Industrial. [2018]. Disponível em: <https://gru.inpi.gov.br/pePI/ssp/patentes/ PatenteSearchBasico.jsp >. Acesso em: jun. 2018.

KUMAR, Pankaj. Alcoholic Beverages Market Expected to Reach \$1,594 Billion, Globally, by 2022 - Allied Market Research. 2017. Disponível em: < https://www.prnewswire.com/newsreleases/alcoholic-beverages-market-expected-to-reach-1594-billion-globally-by-2022---allied-marketresearch-618354513.html>. Acesso em: 6 jul. 2018.

LIMA, Larisse Araújo et al. Sinopse do Cenário Cervejeiro: o advento da produção e o mercado na Região Centro Oeste. Cadernos de Prospecção, Salvador, v. 10, n. 4, p. 650-664, 2017. Disponível em: <https://portalseer.ufba.br/index.php/nit/article/view/2304>. Acesso em: ago. 2018.

MANKINS, John C. Technology readiness assessments: a retrospective. Acta Astronautica, Canadá, v. 65, n. 9-10, p. 1216-1223, nov. 2009. Disponível em: <http://dx.doi.org/10.1016/j. actaastro.2009.03.058>. Acesso em: 28 jun. 2018.

MARTIN, Ben R. Foresight in science and technology. Technology Analysis \& Strategic Management, [S.1.], v. 7, n. 2, p. 139-168, 1995. Disponível em: <https://www.tandfonline.com/ doi/abs/10.1080/09537329508524202>. Acesso em: 28 jun. 2018.

MARTINEZ, Marian Garcia; BRIZ, Julian. Innovation in the Spanish food \& drink industry. The International Food and Agribusiness Management Review, [S.1.], v. 3, n. 2, p. 155-176, 2000. Disponível em: <https://www.sciencedirect.com/science/article/pii/S1096750800000331 > . Acesso em: 28 jun. 2018.

MENESES, Roberta Barbosa de et al. Prospecção tecnológica sobre queijo de coalho. Cadernos de Prospecção, Salvador, v. 4, n. 4, p. 43-49, 2011. Disponível em: <https://portalseer.ufba.br/index. php/nit/article/view/11519>. Acesso em: 28 jun. 2018.

PANZOLINI, Carolina Raquel Leite Diniz et al. Estudo prospectivo sobre tecnologia desenvolvida para obtenção de produtos à base de quinoa para a indústria alimentícia. Cadernos de

Prospecção, Salvador, v. 10, n. 4, p. 765-775, 2017. Disponível em: <https://portalseer.ufba.br/ index.php/nit/article/view/22957>. Acesso em: 28 jun. 2018.

PINHEIRO, Jaqueline Silva Nascimento et al. Estudo prospectivo relativo à atividade da planta babaçu para cosméticos e alimentos. Cadernos de Prospecção, Salvador, v. 8, n. 2, p. 348-354, 2015. Disponível em: <https://portalseer.ufba.br/index.php/nit/article/view/11646/pdf_112>. Acesso em: 28 jun. 2018.

QUESTEL INTELLIGENCE. Intellectual Property. 2018. Disponível em: < https://www.questel. com/>. Acesso em: jun. 2018.

QUINTELLA, C. M. et al. Cadeia do biodiesel da bancada à indústria: uma visão geral com prospecção de tarefas e oportunidades para P\&D\&I. Revista Química Nova, Santa Catarina, v. 32, n. 3, p. 793-808, 2009. Disponível em: <http://www.scielo.br/scielo.php?script=sci_arttext\&pid =S0100-40422009000300022>. Acesso em: 28 jun. 2018.

SILVA, Marcio Lima et. al. Prospecção tecnológica de processos e equipamentos para reabilitação fisioterapêutica. Cadernos de Prospecção, Salvador, v. 10, n. 3, p. 541-551, 2017. Disponível em: < https://portalseer.ufba.br/index.php/nit/article/view/23023/23023>. Acesso em: ago. 2018. 
UCHÔA, Silvia Beatriz Beger; CAMINHA, Tainá Cardoso da Silva. Prospecção tecnológica de alimento em forma de barras de cereais utilizando resíduos. Cadernos de Prospecção, Salvador, v. 8, n. 3, p. 552-561, 2015. Disponível em: <https://portalseer.ufba.br/index.php/nit/article/ view/11665>. Acesso em: 28 jun. 2018.

\section{Sobre os autores}

\section{Marcio Lima da Silva}

E-mail: dasilva.marciolima@gmail.com

Doutor em Mecânica dos Fluidos, Energética e Processos, pela Universidade de Rhones-Alpes, França. Engenheiro Mecânico pela Universidade de Brasília (UnB).

Endereço profissional: Universidade de Brasília. Centro de Apoio ao Desenvolvimento Tecnológico - CDT/UnB. Brasília, DF.

\section{Larisse Araújo Lima}

E-mail: larissealima@gmail.com

Mestre em Ciências de Materiais Nanoestruturados e graduada em Química, pela Universidade de Brasília (UnB). Endereço profissional: Universidade de Brasília. Centro de Apoio ao Desenvolvimento Tecnológico - CDT/UnB. Brasília, DF.

\section{Lincoln Pinheiro Oliveira}

E-mail: pinheiro.lincoln@hotmail.com Mestre em Tecnologias Química e Biológica, pela Universidade de Brasília (UnB) e engenheiro químico, pela UFVJM.

Endereço profissional: Universidade de Brasília. Centro de Apoio ao Desenvolvimento Tecnológico - CDT/UnB. Brasília, DF.

\section{Luiza Xavier da Silva Tenório}

E-mail: luiza.xavier.st@gmail.com Mestre em Zoologia e graduada em Ciências Biológicas pela Universidade de Brasília (UnB).

Endereço profissional: Universidade de Brasília. Centro de Apoio ao Desenvolvimento Tecnológico - CDT/UnB. Brasília, DF.

\section{Sarah Sampaio Py-Daniel}

E-mail: sarahpydaniel@gmail.com Mestre em Ecologia e graduada em Ciências Biológicas com ênfase em Biodiversidade e Conservação.

Endereço profissional: Universidade de Brasília. Centro de Apoio ao Desenvolvimento Tecnológico - CDT/UnB. Brasília, DF.

\section{Thiago Lara Fernandes}

E-mail: thiagolarafernandes@hotmail.com Graduado em Química pela Universidade Católica de Brasília (PUC-Brasília).

Endereço profissional: Universidade de Brasília. Centro de Apoio ao Desenvolvimento Tecnológico - CDT/UnB. Brasília, DF. 


\section{Grace Ferreira Ghesti}

E-mail: ghesti.grace@gmail.com

Doutora em Química pela Universidade de Brasília (UnB). Mestra, pelo Programa de Mestrado Profissionalizante em Certified Brewmaster Course Versuchs- und Lehranstalt für Brauerei in Berlin, VLB, Alemanha. Mestra em Química pela Universidade de Brasília (UnB). Graduada em Química (Bacharelado) pela Universidade de Brasília (UnB).

Endereço profissional: Universidade de Brasília. Instituto de Química - IQ/UnB. Brasília, DF. 